\title{
Privacy-Preserving Hypothesis Testing for Reduced Cancer Risk on Daily Physical Activity
}

\author{
Hiroaki Kikuchi ${ }^{1} \cdot$ Xuping Huang $^{2} \cdot$ Shigeta Ikuji $^{3}$ - Manami Inoue ${ }^{4}$
}

Received: 11 October 2017 / Accepted: 4 March 2018 / Published online: 4 April 2018

(C) The Author(s) 2018

\begin{abstract}
Privacy preserving data mining for medical information is an important issue to guarantee confidentiality of integrated multiple data sets. In this paper, we propose a secured scheme to estimate related risk of cancers accurately and effectively in a privacy-preserving way. We study models to configure the appropriate set of attributes to reduce risk of identity of an individual from being determined. We examine the proposed privacy preserving protocol for encrypted hypothesis test, using actual cohort data supplied by National Cancer Center.
\end{abstract}

Keywords Privacy $\cdot$ Privacy-preserving data mining $\cdot$ Epidemiology $\cdot$ Hypothesis testing

\section{Introduction}

\section{Background}

Risk factors for cancers have been widely investigated in conventional works. For examples, Cardis et al. [1] at International Agency for Research on Cancer carried out collaborative studies of cancer risks after low doses of

This article is part of the Topical Collection on New Technologies and Bio-inspired Approaches for Medical Data Analysis and Semantic Interpretation

\footnotetext{
$\triangle$ Hiroaki Kikuchi

kikn@meiji.ac.jp

Xuping Huang

huang_xp@meiji.ac.jp

Shigeta Ikuji

shigeta.ikuji@access-company.com

Manami Inoue

mnminoue@ncc.go.jp

1 School of Interdisciplinary Mathematical Sciences, Meiji University, 4-21-1 Nakano, Tokyo 164-8525, Japan

2 Strategic Coordination of Research and Intellectual Properties, Meiji University, 4-21-1 Nakano, Tokyo 164-8525, Japan

3 ACCESS CO., LTD., 3 Neribei, Kanda, Chiyoda, Tokyo 101-0022, Japan

4 Center for Public Health Sciences, National Cancer Center, 5-1-1 Tsukiji, Chuo-ku, Tokyo 104-0045, Japan
}

ionizing radiation among nearly 600,000 radiation works in the nuclear industry in 15 countries. The result indicates the excess relative risk for cancers other than leukemia was 0.97 per Sv, $95 \%$ confidence interval 0.14 to 1.97 . They also figure out that excess risk of cancer exists for nuclear workers even at the low doses and dose rate.

However, during these studies of cancers, confidentiality and criticality of privacy information should be considered because of exposure of cancers. For big data mining, integration of multiple data collected via ubiquitous sensors, smart-phones, and portable devices makes epidemiological study more accurate. To achieve accurate data processing as well as privacy preserving, we consider the issues as follows:

privacy issues of patients Given in a confidential dataset, even for medical studies, no cancer patients want to be exposed due to privacy concerns.

inconsistent identities in multiple datasets Proprietary identifiers are used to identify individuals. However, in case of integration of multiple datasets, it is difficult to assume a global identity. In case of integrated datasets with inconsistent identifiers, finding alternatives of identities has been a challenge.

A set of personal attributes, which is used to identify individuals, includes name, address, and telephone numbers, etc. However, models should be studied to configure appropriate set of attributes, especially optimal combination of attributes because of the unavailability of uniqueness of personal attributes. 


\section{Related works}

In the conventional works, statistical inference applied to single or multiple datasets has been proposed in many works. Privacy preserving algorithm is required because of the possibility of identifying individual participants by publicly available aggregate statistics, pointed out by Homer et al. [2]. Statistical estimator is studied by Smith [3] and Rakesh et al. [4] and risk-utility has been discussed in Fienberg et al. [5]. Binary hypothesis testing under privacy constraints for large datasets has been studied in Liao et al. [6]. Privacy preserving protocol for radiation data and partitioned data are discussed by Kikuchi, et al. [7] and Vaidya et al. [8].

In order to figure out features of medical data and to clarify correlation between different parameters in a dataset or causal correlation between attributes from different datasets, hypothesis testing supplies an effective statistical inference to determine distribution in a certain dataset, however, in most of conventional works, raw data is used for analysis.

In this work, we propose a new private preserving hypothesis testing protocol, as well as specifying the best combination of significant personal attributions as the quasiidentifier to identify particular user in multiple datasets for statistics inference.

\section{Our contributions}

In this paper, we propose privacy-preserving schemes to estimate the relative risk (RR) of cancers, using the cryptographic protocol Private Set Intersections (PSI) to achieve secured epidemiological processing including set intersection for mortality rate, and evaluation of test statistics for hypothesis testing. Confidentiality of data is preserved even after intersection of two subsets.

Our contributions of are listed as follows:

- propose a privacy-preserving protocol for hypothesis testing using a set of personal attributes as quasi-identifiers

- take an experiment of the proposed protocol to estimate relative risk of cancer in terms of quantity daily physical activities

\section{Privacy-preserving hypothesis testing}

\section{Purpose statement}

In this paper, we consider a use case of data analysis toward distributed datasets supplied by different providers.

In case of risk of radiation, suppose party $A$ be an agency which maintains lists of workers who are exposed to dose of radiation. This kind of data is available since there are regulations specifying the limit of total annual dose of radiation and employees in nuclear-power stations are supposed to declare the record of dose of radiation in many countries. In Japan, working under more than $50 \mathrm{mSv}$ is prohibited $[9,10]$. Party $B$ is a hospital for cancers and keeps a dataset of cancer patients.

Both of parties $A$ and $B$ should keep their datasets $X_{A}$ and $X_{B}$ confidential, however, correlation between the risk of cancer and dose of radiation should be contributive and useful for further medical care and research. Thus, a privacy-preserving scheme for confidential computing for distributed dataset is required.

Death rates or mortality rate for both datasets are compared to clarify the correlation. The mortality rate is adjusted for different distributions of age groups in both of datasets. Let $X_{A, y}$ be a subset of $X_{A}$ with increments of 10 years. Then $X_{A}$ can be partitioned as $X_{A}=X_{A, 30} \cup X_{A, 40} \cup$ $\ldots \cup X_{A, 80}$. The expected numbers of subjects to death can be known as standardized mortality rate.

\section{Relative risk}

We examine the risk factors for a disease by dividing participants into two groups with and without exposure in a cohort study. The relative risk is defined as the ratio of a diseased member receiving exposure to a diseased member without receiving exposure [12].

Table 1 shows a contingency table for a case-control study for cancer as an example, which is a $2 \times 2$ table of observed frequencies with a sample size $N$. In Table 1, $m_{1}$ represents smoking participants and $m_{2}$ represents nonsmoking participants. $a$ and $b$ suffer from cancer, while $c$ and $d$ do not during the investigation. Then the $R R$ of smoking is defined as the probability of diseased (cancer) participants in the exposed (smoking) group out of the probability of diseased participants in the unexposed (nonsmoking) group as follows.

$$
\begin{aligned}
R R & =\frac{\operatorname{Pr}(\text { cancer } \mid \text { smoking })}{\operatorname{Pr}(\text { cancer } \mid \text { non-smoking })} \\
& =\frac{a}{n_{1}} / \frac{c}{n_{2}}=\frac{a(c+d)}{(a+b) c} \approx \frac{a d}{b c}
\end{aligned}
$$

A RR greater than 1.0 indicates an increased risk of disease in exposed group. Hypothesis test is used to examine the confidence of RR in this paper as follows:

Table 1 Contingency table for a case-control study

\begin{tabular}{llll}
\hline & Smoking & Non-smoking & Total \\
\hline Cancer & $a$ & $b$ & $n_{1}$ \\
Noncancer & $c$ & $d$ & $n_{2}$ \\
Total & $m_{1}$ & $m_{2}$ & $N$ \\
\hline
\end{tabular}


null hypothesis: $H_{0}$ : The proportion of participants who suffer from cancer equals between smoking participants and non-smoking participants;

alternative hypothesis: $H_{A}$ : The proportion of smoking participants who suffer from cancer differs from nonsmoking participants;

Given the null hypothesis $H_{0}$, the expected value $E_{1}$ is calculated by multiplying two independent probabilities for each cell of the contingency table that $\operatorname{Pr}$ (cancer) $=n_{1} / N$ and $\operatorname{Pr}$ (smoking) $=m_{1} / N$, as follows:

$E_{1}=\frac{n_{1}}{N} \frac{m_{1}}{N} N=\frac{n_{1} m_{1}}{N}$.

We compare the difference between observed frequencies $O_{i}$ and the expected frequencies $E_{i}$ in each category of the $2 \times 2$ cells contingency table using chi-squared test. The probability distribution $\chi^{2}$ is computed as follows:

$$
\begin{aligned}
\chi^{2} & =\sum_{i=1}^{2 \times 2} \frac{\left(\left|O_{i}-E_{i}\right|-1 / 2\right)^{2}}{E_{i}}, \\
& =\frac{N(|a d-b c| \pm N / 2)^{2}}{n_{1} n_{2} m_{1} m_{2}} .
\end{aligned}
$$

Where, $\chi^{2}$ is approximated by a chi-squared distribution with $(2-1)(2-1)$ degrees of freedom. Given a chi-squared distribution with one degree of freedom, the outcome of $\chi_{1}^{2}=3.84$ cuts off the upper $5 \%$ of the tail of the distribution.

Alternatively, if employ $\chi$ is with a normal distribution $N(0,1)$, we can test whether

$\chi=\frac{\sqrt{N-1}\{(a d-b c) \pm N / 2\}}{\sqrt{n_{1} n_{2} m_{1} m_{2}}}$,

is less than $Z(0.05 / 2)=1.960$ with $95 \%$ confidence.

\section{Private set intersection}

Private set intersection (PSI) is a cryptographic protocol which allows multiple parties to compute the intersection of their private sets without revealing anything about their sets. A number of protocols have been proposed so far after Freedman, Nissim and Pinkas proposed the first PSI protocol using polynomial expression of sets in [17]. Abadi et. al showed the delegated PSI protocol on outsourced private datasets, which assumes the use in cloud data store in [18]. Among then, the following three works can be used to estimate the size of intersection $|X \cap Y|$, which corresponds to the population of smoker $(X)$ suffering from cancer $(Y)$.

FNP04 (oblivious polynomial evaluation) [17] The scheme presented in [17] uses oblivious polynomial evaluation in which elements of set are represented as polynomials $f(x)$ over a finite field. It is a two-party protocol with one party encoding its elements $x_{1}, x_{2}, \ldots$ as the roots of the polynomial and the other party evaluating $f\left(y_{1}\right), f\left(y_{2}\right), \ldots$ in a privacy preserving way. The evaluation of the polynomial turns to be 0 if and only if $x_{i}=y_{j} \in X \cap Y$. The drawback of the protocol is the computational complexity. The running cost is proportional to the order of polynomial which equals to the number of elements of the set.

SSP (Secure Scalar Product) [16] Scalar product of two vectors is performed securely using an additive homomorphic public-key algorithm. It is a two-party protocol which can be used by many applications as one of primary building block. The many instances of additive homomorphic algorithms include Paillier encryption [14], Lattice-based encryption, and elliptic-curve cryptosystem. The fundamental version allows vectors of arbitrary values. While, the set intersection requires Boolean vector consisting of 1 or 0 value indicating membership of subsets. Hence, it is too expensive (in terms of computational cost) to evaluate the size of set intersection in privacy preserving manner.

AES03 (commutative one-way function) [13] Agrawal, et. al. used a commutative Pohlig-Hellman cipher [15] and secure hash function as building blocks to construct twoparty protocol to compute set intersection. As one of the extension, they also showed the modified protocol that obtains only the size of intersection without seeing the elements of the intersection. The idea of their protocol is that commutative property of two independent encryptions allows to figure out the common element of two subsets. Let $f$ and $g$ be (symmetric but commutative) encryption privately generated by Alice and Bob, respectively. A common element $x$ can be identified by testing that $f(g(a))=g(f(a))$ because of the commutative property of encryptions.

We show Algorithm 1 that replaces commutative encryptions $f$ and $g$ by Pohling-Hellman cipher, defined simply as $f(m)=H(m)^{u} \bmod p$ and $g(m)=H(m)^{v} \bmod$ $p$, respectively. Note that the algorithm is the version of size of intersection only and can be used to determine the elements of intersection by modifying the Step 2 to send the $\left(H(x)^{u}\right)^{v}$ together with the ciphertext $H(x)^{u}$.

Algorithm runs efficiently when the subset is small in the domain of values. Suppose that we have a set of integers ranging from 0 to 100 and subsets of 30 elements for each. In SSP protocol, both parties need iterate public-key encryptions for all possible elements of domain, i.e., 100 times. On the other hand, AES and FNP protocols performs as many as the size of subset, i.e., 30 times. FNP requires polynomial evaluation that runs squared times of the order of polynomial, resulting $30^{2}=900$ computations. Hence, AES has the least computational costs of 30 times and is 
most appropriate for the application that the intersection is small enough to the domain.

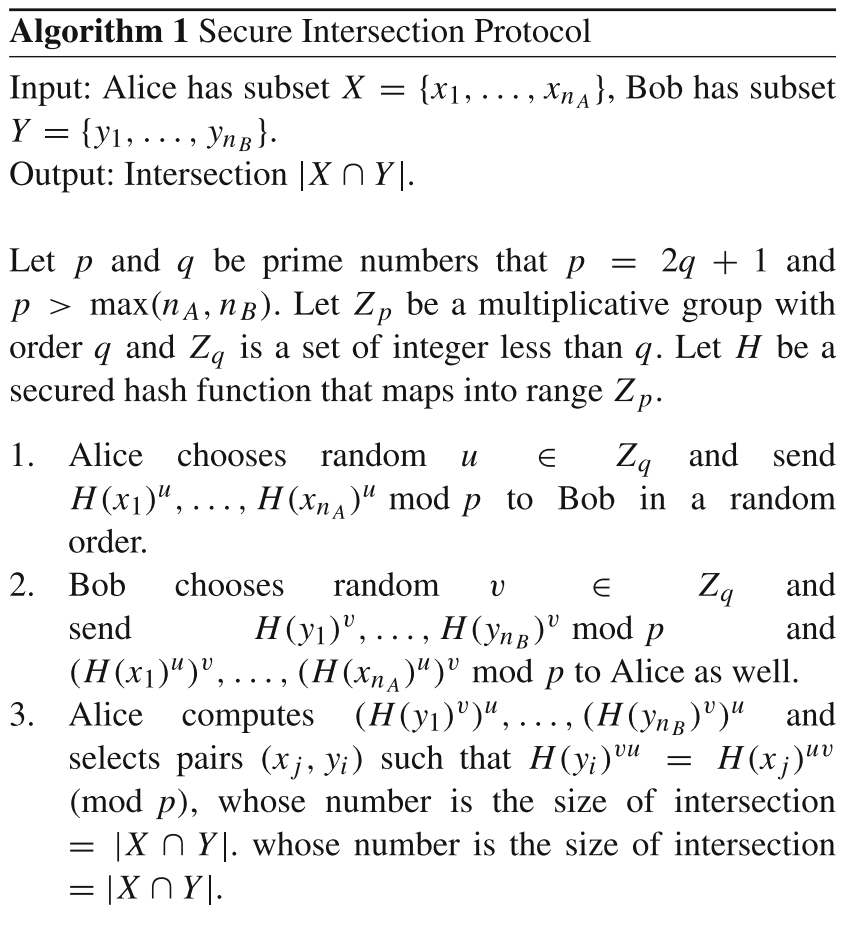

\section{Hypothesis testing}

To model discrete events which occurs infrequently in time series, such as cancer and death, Poisson distribution is widely used. Let $X(X \in \mathbb{N} \cup\{0\})$ be a random variable that represents the number of occurrences of some events over a given time interval. Let $\lambda(\lambda>0)$ be a constant that denotes the average number of events in an interval. If the probability that $X$ assumes $k$ is

$P(X=k)=\frac{e^{-\lambda} \lambda^{k}}{k !}$,

then $X$ is said to have a Poisson distribution with the rate parameter $\lambda$.

Suppose we observe $X=O$ deaths given $E$ as the expected number of deaths. We consider a Standardized Mortality Ratio (SMR) defined by

$S M R=\frac{O}{E}=\frac{\sum_{j} d_{j}}{\sum q_{j} n_{j}}$,

where $d_{j}$ is the observed number of deaths at the $j$-th age interval in the interested condition to be tested, $n_{j}$ is the general population at $j$-th age interval and $q_{j}$ is the mean death rate in the $j$-th group. Note that $q_{j}$ times $n_{j}$ gives the expected number of deaths at $j$-th age interval.

We wish to determine whether the SMR is close to 1 or not. Namely, if the SMR in workers in nuclear-power station is equal to that of ordinary SMR, the risk of radiation is not significant. Hence, we test null hypothesis

$H_{0}: \lambda=E$

against the alternative hypothesis

$H_{1}: \lambda \neq E$.

If we conduct a one-sided test,

$$
\begin{aligned}
p & =P(O \mid E)+P(O+1 \mid E)+\ldots, \\
& =1-\sum_{j=0}^{O-1} \frac{E^{j}}{j !} e^{-E}
\end{aligned}
$$

gives $p$-value of the test. Employing approximation of Poison distribution when $E \geq 5$, the test statistic

$Z=\frac{O-E \pm 0.5}{\sqrt{E}}$

has an normal distribution $N(0,1)$ with mean 0 and the standard deviation 1 . Note that 0.5 is the constant. If we conduct a two-sided test, the test statistic satisfying

$Z=\frac{|O-E|-0.5}{\sqrt{E}}>Z(\alpha / 2)$

would reject the null hypothesis at the $\alpha$ level of significance.

\section{Privacy search oracle model}

Personal attribute may be used to identify individuals. The accuracy of identification depends on type of attributes. For example, attribute sex is 1-bit information to classify the set of individuals into two classes. Birthday has a domain of 365 ways, which is equivalent to $\log _{2}(365)=8.51$ bit entropy. Hence, combining sex and birthday could be $1+8.51=9.51$ bit entropy, which could identify $2^{9.51}=$ 729 individuals in average.

Yasui et. al study some sets of personal attributes in term of entropy in [11]. They proposed Privacy Search Oracle model to quantify the information of attribute based on the statistics of Social Network Services. The population of $120,000,000$ individuals is identified uniquely with set of attributes of 27-bit entropy $\left(2^{27}=134,000,000>\right.$ $120,000,000)$. Table 2 summaries the entropies of some subsets of personal attribute as well as the ambiguity level.

\section{Privacy-preserved estimation of reduced cancer risk}

\section{Problem description}

Consider Alice is a national cancer center that maintains comprehensive personal attributes for patients of gastric cancer, lung, colon, and so on. In our study, we mainly focus 
Table 2 Entropy of personal attributes

\begin{tabular}{|c|c|c|c|c|}
\hline Personal & Entropy [bit] & Ambiguity & Max \# of & Description \\
\hline Attribute & & & Duplicated IDs & \\
\hline Name in Chinese char. (Kanji) & 27 & High & 24 & Same name can be written in several ways. \\
\hline Name in Japanese char. (Kana) & N/A & Low & 30 & Several representations can be unified. \\
\hline Sex & 1 & None & 61,020 & Male or female (1 bit) \\
\hline Birthday and year & 15 & None & 86 & 365 days (15 bit) \\
\hline Mailing address & 26 & Low & 56 & Almost unique but several representations in font. \\
\hline City (ku, machi, mura) & 14 & High & 12,131 & Not very unique for historical reason. \\
\hline Prefecture (states) & 6 & None & 22,336 & Unique. \\
\hline $\mathrm{C} / \mathrm{A}$ & 2 & High & N/A & Occasionally specified. not complete attribute. \\
\hline
\end{tabular}

on colon cancer because the risk of colon cancer has been known as significantly correlated to daily physical activity. Alice owns the set of colon cancer as $X$.

Bob is a provider for datasets, indicating interests on personal physical activity. Examples include a sport club that records frequency of exercises for each member, a public health center that periodically investigate personal information of citizens, or a commercial health company that monitors daily physical activity quantities from vital devices. Metabolic equivalents (METs) is used to determine quantity daily total physical activity level, based on questionnaires about hours/day in heavy physical works, hours/day in walking, hours/day in sitting, and the days/week in leisure-time sports or exercise [19]. With the METs score, Bob classifies the people into four $(q=4)$ orthogonal classes; Lowest (L), Second (S), Third (T), and Highest $(\mathrm{H})$, specified by four subsets of $U, Y_{1}, Y_{2}, Y_{3}$ and $Y_{4}$, respectively.

\section{Number of people with exact the same names}

Proprietary identifiers are used to identify individuals for institutes who own datasets. However, in order to join multiple datasets with inconsistent identifiers, alternatives of identifies are necessary. We study a set of significant personal attributes to identify unique individual as a quasiidentifier. For example, name attribution is as known as an almost unique attribute, however, there are exceptions that when multiple users have the exact same surname and given name. Figures 1 and 2 shows the population of people in which $x$ individuals have the exact same surname and given name in some datasets: JPHC, ${ }^{1}$ Univac [20], and NTT. $^{2}$ Both of vertical and horizontal axis are plotted in log scale. In JPHC, there are about 100 thousand people with unique name $(x=1)$, which becomes about 2 thousand when two people are with the same name $(x=2)$.

\footnotetext{
${ }^{1}$ the Japan Public Health Center-based Prospective Study (JPHC)

${ }^{2}$ NTT telephone book 2001
}

Figure 1 shows the number of $x$ individuals who have the identical name written in Chinese character (Kanji), and Japanese character (Hiragana).

Based on the observation of the distribution of people with the exact same names, we adapt a mathematical model of Zipf's law, which states that the number of people, $f(x)$, with the $x$-th order is proportional to $1 / x$.

Accordingly, we have the population of $x$ individuals with the same name written in Hiragana as

$f(x)=\frac{a}{x^{s}}=\frac{110000}{x^{3.87}}$

where $s$ is a constant characterized by the given dataset.

A generalized Zipf's model allows to estimate the number of people with the same attribute. The total population $D$ is given by

$D=a \sum_{k=1} \frac{1}{k^{3.87}}$

In Eq. 8, we have the constant $a$. For instance, the number of people with not unique name is given from total population in Japan 120 million as $\left.a=D / \sum_{k=1} \frac{1}{k^{3.87}}\right) \simeq D / 1.1 \simeq$ $109 \mathrm{e}^{6}$. Consequently, we estimate that 109 million people have the same name written in Hiragana. Hence, the name in Hiragana is not significant to identify individuals.

\section{Combination of attributes as a unique identifier}

With our Zipf's model, we quantify the entropy of personal attribute $S$, defined as

$H(S)=\sum_{k} P(k) \log (P(k)) \quad[$ bit/symbol $]$

where $P(k)$ is a probability of symbol $k$, i.e., value of attribute in $S$. Accordingly, the entropies of name in Kanji and in Hiragana in JPHC dataset of 140,420 records are 14.63 and $13.71 \mathrm{bit} / \mathrm{symbols}$, respectively. 
Fig. 1 Distribution of Population for numbers of people written with identical name (in Chinese character, labeled as "Kanji", in Japanese character, labeled as "Kana")

Fig. 2 Distributions of population for numbers of people written with identical name in dataset "JPHC", Univac [20] and NTT

Table 3 Entropies of some combinations of personal attributes

\begin{tabular}{lllll}
\hline Option & Set of attributes & $\begin{array}{l}\text { Entropy } \\
{[\text { bit }]}\end{array}$ & $\begin{array}{l}\text { Max \# } \\
\text { duplicated records }\end{array}$ & $\begin{array}{l}\text { \# of unresolved } \\
\text { records }\end{array}$ \\
\hline$A$ & Name in Kana, sex & 14 & 30 & 30,180 \\
$B$ & Name in Kana, sex, birthday & 30 & 2 & 16 \\
$C$ & Name in Kana, sex, birthday, state & 36 & 2 & 12 \\
$D$ & Name in Kana, sex, birthday, address & 56 & 0 & 0 \\
$E$ & Name in Kana, birthday, address & 55 & 0 & 16 \\
$F$ & Name in Kana, address & 40 & 2 & 10 \\
$G$ & Sex, birthday, address & 42 & 2 & 10 \\
\hline
\end{tabular}
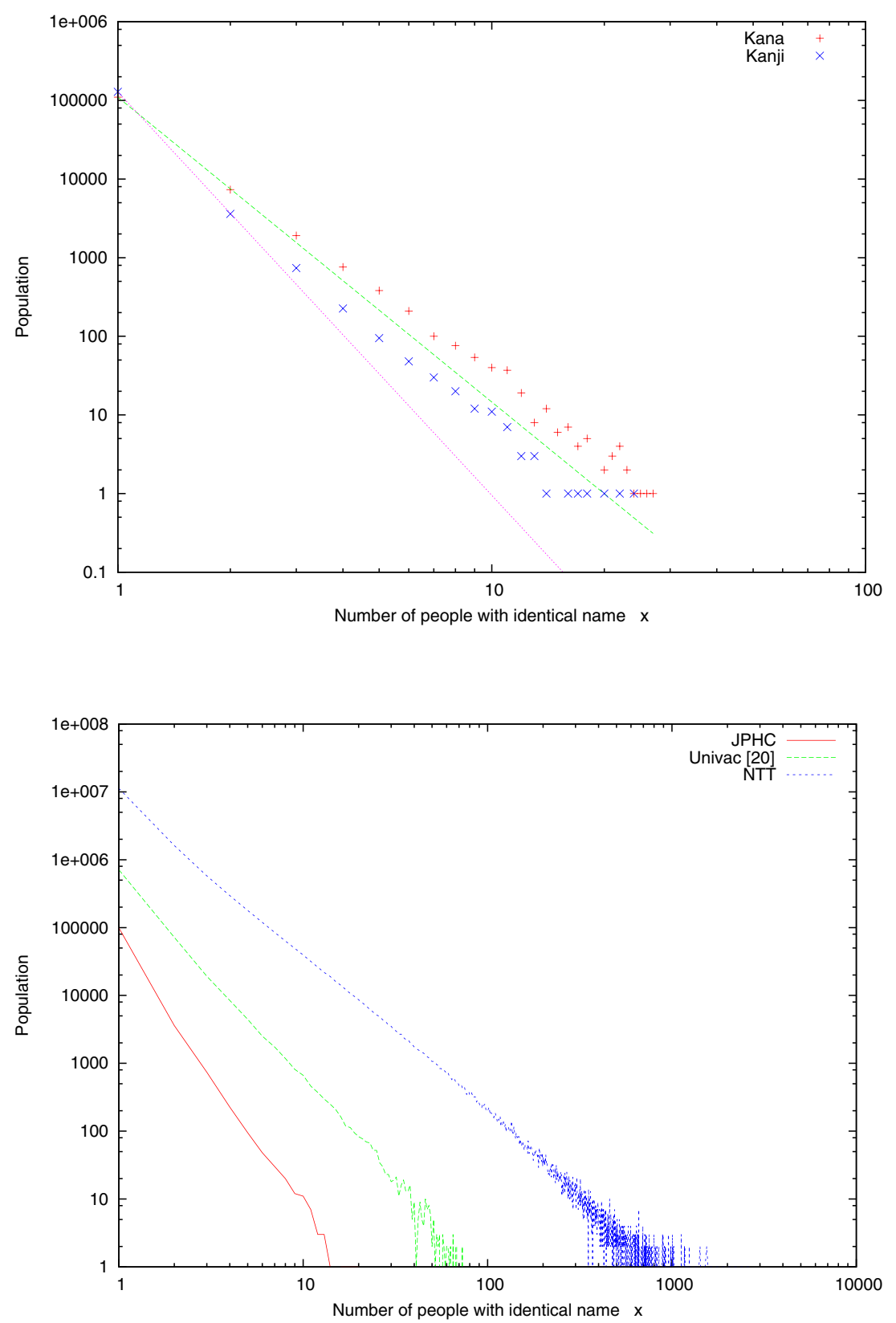
Table 4 Contingency table and relative risks with test statistic

\begin{tabular}{lllllll}
\hline & $\left|X \cap Y_{p}\right|$ & $\left|Y_{p}-\left(X \cap Y_{p}\right)\right|$ & $Y_{p}$ & $\mathrm{RR}$ & $N_{p}$ & $\chi_{p}^{2}$ \\
\hline$Y_{1}$ & $c$ & $d$ & $c+d$ & 1.0 & - & Reference \\
$Y_{2}$ & $a_{2}$ & $b_{2}$ & $a_{2}+b_{2}$ & $\frac{a_{2}}{a_{2}+b_{2}} / \frac{c}{c+d}$ & $a_{2}+b_{2}+c+d$ & $\frac{N_{2}\left(a_{2} d-b_{2} c\right)^{2}}{\left(a_{2}+b_{2}\right)(c+d)\left(a_{2}+c\right)\left(b_{2}+d\right)}$ \\
$\vdots$ & $\vdots$ & $\vdots$ & $\vdots$ & $\vdots$ & $\vdots$ \\
$Y_{q}$ & $a_{q}$ & $b_{q}$ & $a_{q}+b_{q}$ & $\frac{a_{q}}{a_{q}+b_{q}} / \frac{c}{c+d}$ & $a_{q}+b_{q}+c+d$ & $\frac{N_{q}\left(a_{q} d-b_{q} c\right)^{2}}{\left(a_{q}+b_{q}\right)(c+d)\left(a_{q}+c\right)\left(b_{q}+d\right)}$ \\
\hline
\end{tabular}

We examine the JPHC dataset with 111,458 records in the same way. ${ }^{3}$ Table 3 shows the number of duplicated (more than two) records for some combinations of personal attributes. For example, a combination of name written in Hiragana and sex is used as the quasi-identifier as option $A$, however, there are 30,180 records which cannot be uniquely identified because more than two records share the exactly the same name and sex. The most common name is shared by 30 individuals.

According to Table 3, we find options $D$ (name, sex, birthday, address) and $E$ (name, birthday, address) uniquely identify all individuals in JPHC dataset.

\section{Proposed scheme}

Since identities used by Alice are not consistent with that used by Bob, which considering the combination of multiple datasets, instead of proprietary identities, we use a combination of significant personal attributes, such as names in Hiragana and birthday, as quasi-identifiers, which can be computed using secure hash function, e.g., SHA256, as

$i=\operatorname{Hash}$ (name $\|$ birthday $\|$ address)

where $\|$ is a symbol of concatenation. A person who belongs to both datasets $A$ and $B$ is uniquely identified by the quasi-identifier defined over $U$ as the range of secure hash function.

We propose a cryptographic protocol between Alice with $X$ and Bob with $Y_{1}, \ldots, Y_{q}$ for privacy-preserved relative risk estimation without revealing identities to other parties in Algorithm 2. It uses Algorithm 1 as a sub-protocol.

It aims to compute relative risks in terms of some interested attributes. It is a two-party cryptographic protocol of a party (Alice) with set of (colon cancer) patients and a party (Bob) with questionnaire survey results of patients. Alice and Bob could be the national registry of cancer and local government that conduct user study of citizens. Both parties are not allowed to share the database without consent of all subjects. Instead of sharing, Algorithm 2 allows them to evaluate relative risks of cancer in terms of questionnaire survey without revealing who suffered

${ }^{3}$ It excludes the missing records in some attribute. cancers or the survey results. In our experiment, we are interested in clarifying relative risk of colon cancer in terms of sex and the frequency of daily physical activities. Algorithm 2 also gives the test statistics $\chi$ to perform hypothesis testing without revealing any personal attribute.

Algorithm 2 runs efficiently even with the big-data studies because the computational cost is not depending on the whole domain size but on the size of interested subsets.

Algorithm 2 Privacy-Preserving Relative Risk Estimation for $q \times 2$-contingency table

Input: $\quad$ Alice has target subset $X$ of a set of all identities $U$. Bob has $q$ attribute subsets $Y_{1}, Y_{2}, \ldots, Y_{q}$ of $U$, where $Y_{1}, \ldots, Y_{q}$ are partition of $U$, i.e., $Y_{1} \cup Y_{2} \cup \cdots \cup Y_{q}=U$ and $Y_{i} \cap Y_{j}=\varphi$ for all $i \neq j$.

Output: relative risks $R R_{1} \ldots, R R_{q}$ of $q$ attributes for target attribute $X$

Step 1. Alice and Bob use Algorithm 1 to compute $c=$ $\left|X \cap Y_{1}\right|$ and

$a_{i}=\left|X \cap Y_{i}\right|$

for $i=2, \ldots, q$.

Step 2. Given $c$ and $a_{i}$, Alice computes $d=\left|Y_{1}\right|-c$,

$$
b_{i}=\left|Y_{i}\right|-a_{i}
$$

for $i=2, \ldots, q$.

Step 3. Alice computes relative risks $R R_{2}, \ldots, R R_{q}$ and the corresponding $\chi_{2}^{2}, \ldots, \chi_{q}^{2}$ according to Table 4 .

\section{Experimental evaluation}

\section{Experiment with JPHC dataset}

We implemented the proposed protocol and applied it to JPHC Dataset with 99,127 individuals. Table 5 shows the experimental results. For men's third METs class $(T)$, the test statistic is $\chi_{T}^{2}=6.54$. For chi-square distribution of 1 degree of freedom, since the probability $p<0.025$ and hence $H_{0}$ is rejected. Therefore, daily physical activities in $T$, and $H$ for men's reduces the relative risk of cancer with significant level of confidence. 
Table 5 Relative risk of colon cancer according to daily total physical activity level

\begin{tabular}{llllll}
\hline \multicolumn{1}{c}{$X$} & $\left|Y_{i}-\left(X \cap Y_{i}\right)\right|$ & $\left|Y_{p}\right|$ & $R R$ & $\chi_{(i)}^{2}$ \\
\hline Men $n=46,236$ & & & & \\
& $(178)$ & $(41,108)$ & $(41,286)$ & & \\
$L(16,374)$ & 79 & 13915 & 13994 & 1.00 & Reference \\
$S(9,594)$ & 36 & 8229 & 8265 & 0.77 & 1.68 \\
$T(9,085)$ & 25 & 7865 & 7890 & 0.56 & 6.54 \\
$H(11,184)$ & 32 & 9830 & 9862 & 0.57 & 7.20 \\
Women $n=52,891$ & & & & \\
& $(130)$ & $(46,330)$ & $(46,460)$ & & \\
$L(17,404)$ & 40 & 14347 & 14387 & 1.00 & Reference \\
$S(13,795)$ & 32 & 11703 & 11735 & 0.98 & 0.01 \\
$T(11,865)$ & 32 & 10283 & 10315 & 1.12 & 0.21 \\
$H(9,827)$ & 19 & 8473 & 8492 & 0.80 & 0.61 \\
\hline
\end{tabular}

However, according to the experimental result, test statistics for women are not significant. The possible reason why the METs scores for women are not significant can be assumed that the distribution of ages was skewed, or the other exposure factors such as smoking habit effects.

We compare our result in Fig. 3 to the existing results in [19] in Fig. 4. According to the results, our proposed privacy-preserving protocol achieved a similar behavior correlation between cancer risk and daily physical activities to the conventional work [19] using raw data. Furthermore, the conventional work evaluated relative risk with hazard ratio or odds ratio, while our results are approached more easily in a privacy-preserving way since when dealing with a rare disease, the relative risk can be approximated by the odds ratio.

\section{Evaluation on performance}

We implement and evaluate the performance of the proposed algorithm with JPHC dataset consisting of 140,000 individuals. Table 6 shows the experimental environment to evaluate the performance. Figures 5 and 6 show processing time according to different size of datasets with $10 \mathrm{k}, 35 \mathrm{k}, 70 \mathrm{k}, 140 \mathrm{k}$ records. We iterate experiments each for 10 times and record the average processing time.

Performance requires a dominant resource for calculation when the modular exponentiation is over than 140k individuals. However, this problem can be solved and the performance can be improved by a distributed computing with multiple machines or parallel computation.

\section{Evaluation on security}

In [13], assuming the random oracle model has no hash collisions, and in semi-honest model, there is no polynomial-time algorithm that can distinguish a random value from $H(x)^{u}$ given $x$.

Summarily, in our use case, data providers are assumed to be honest-but-curious, which is known as the semihonest model, that providers own private datasets following protocols properly but trying to learn additional information about the datasets from received messages. It is rational to assume honest-but-curious model because either party may be interested in learning the personal data so that personal data such as name with disease could be dealt in underground market. Malicious model is too strong to
Fig. 3 Relative risk of colon cancer for METs estimated in the proposed algorithm

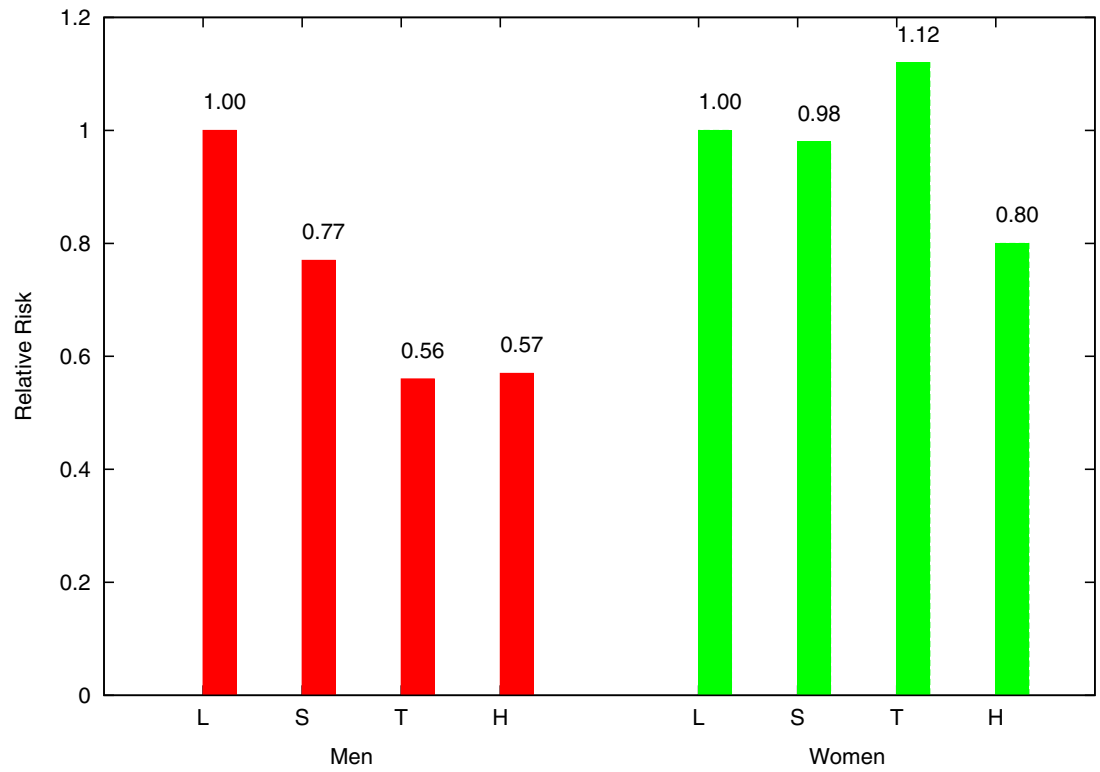


Fig. 4 Relative risk of colon cancer for METs estimated in [19]

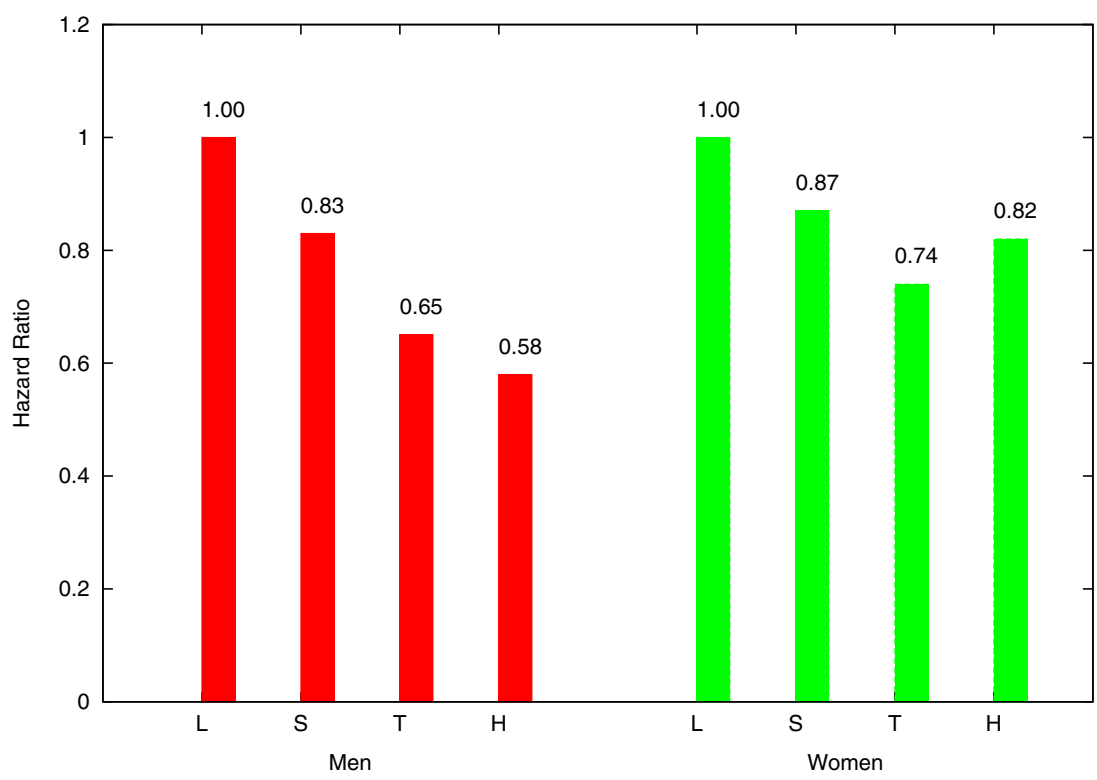

assume in two-party case where the malicious party should be excluded easily.

We make the following remarks about the security of the proposed scheme.

Remark 1 Assuming Decisional Diffie-Hellman hypothesis (DDH), no party learns any element that does not belong to the intersection from the output of Algorithm 1.

Proof DDH claims for any element $\operatorname{gin} Z_{q}$, the distribution of $\left\langle g^{a}, g^{b}, g^{a b}\right\rangle$ is indistinguishable from the distribution of langleg $\left.{ }^{a}, g^{b}, g^{c}\right\rangle$, where $a, b, c \in Z_{q-1}$. Without loss of generality, assume Alice determines $H(y)^{v}$ for any $y \notin Y-$ $-X$ when she has $H(x)^{u}$ and $H(x)^{u v}$ for some known $x$ and $u$. By replacing $g^{a}=H(x)^{u}$ and $g^{a b}=H(x)^{u v}$, she can distinguish $g^{a b}$ with $g^{c}$ because she distinguishes $H(x)^{u v}$ with $H(y)^{v}$ from the above assumption. This contradicts to the DDH. Therefore, we have the proof.

Table 6 Experimental environments

\begin{tabular}{ll}
\hline Modulus size $|p|$ & 2048 bit \\
Order of $G$ & 160 bit \\
Domain of $u, v$ & 160 bit \\
Application impl. & Scala \\
SHA-1 & Java sphlib \\
Modulo & Java Big integer \\
Data Structure & Java HashSet Collection \\
OS & Ubuntu 12.10 amd64 \\
CPU & Intel Celeron Processor G1610 \\
Memory & 4 GB (DDR3 SDRM PC3-10600) \\
Network speed & 46 Mbps (measured values average) \\
\hline
\end{tabular}

Remark 2 No party learns any element that does not belong to any intersections for $q$ subsets.

Proof It is straightforward from the construction of Algorithm 2. Given $c=\left|X \cap Y_{1}\right|$, there are $\left|Y_{1}\right|-c$ possible elements in $Y_{1}$, which are impossible to guess with trivial probability $1 /\left(\left|Y_{1}\right|-c\right)$. Similarly, no information can be learned from $a_{i}=\left|X \cap Y_{i}\right|, b_{i}=\left|Y_{i}\right|-a_{i}$ for $i=2, \ldots, q$.

The threat is the risk that malicious parties may figure out the particular individual depending on the chance to identify random numbers in the algorithm. The probability to pick the correct random number is

$S=\frac{1}{|u|}=2^{-160}$,

which is almost infeasible. Thus, the proposed scheme is secure against the malicious party to guess the individual.

\section{Comparison to conventional works}

This paper proposed a privacy-preserving hypothesis testing, which is the novelty of this paper and not achieved by conventional works. Thus, direct comparison is difficult. Instead, we show the relationship between possible building blocks and the proposed two schemes in Table 7. The proposed scheme performs about 360 element per second that is estimated based on the trial implementation. Our scheme performs better than any other protocol based on SSR and FNP because the computational complexity is proportional to the number of element in the subsets. 
Fig. 5 Processing time for dataset size in Alice

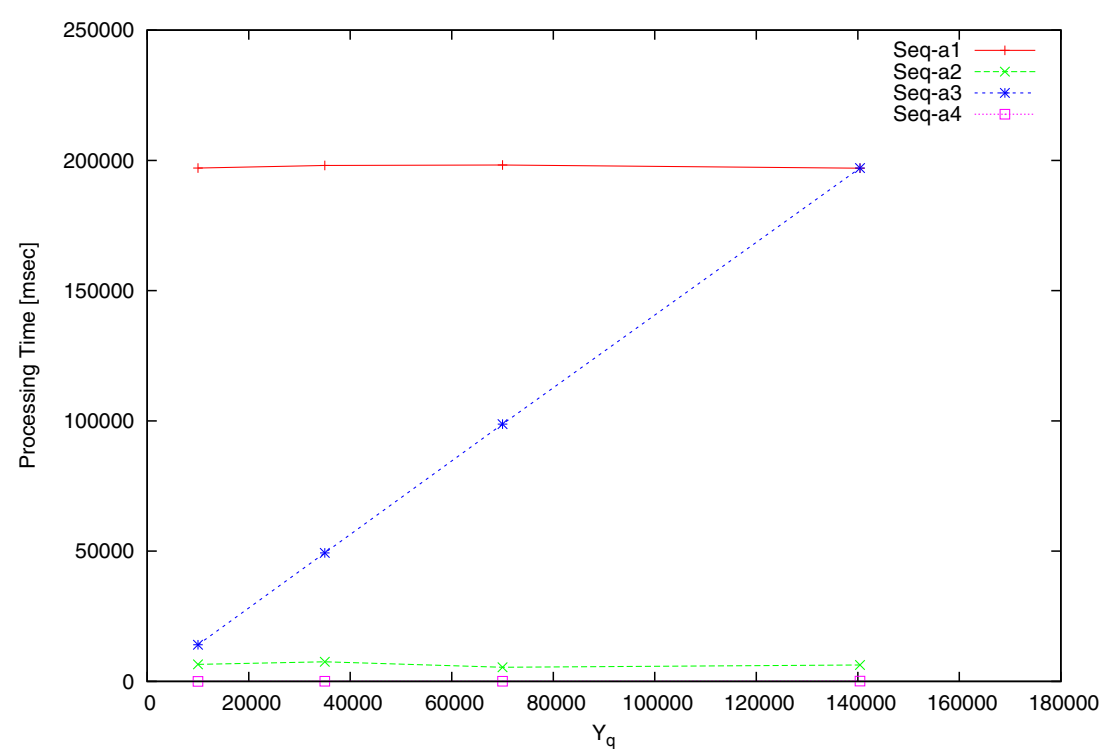

\section{Other applications}

PSI protocol is applicable to broad fields not only in cancer risk evaluation but also in enterprise and government. We give some potential applications.

\section{Intellectual property and patents}

Enterprise having confidential technologies are interested to seek their competitor's patents. However, it is not clear if their competitor owns unpublished intellectual property that conflict with its private technology. If they share the common technologies, they would like to collaborate or to license each other. If their confidential technologies are disjoint, they want to keep that secret. This can be solved by applying PSI protocol with the set of technical terms of documents. They could make sure whether their unpublished intellectual properties are common or disjoint without revealing confidential document.

\section{Epidemiological studies}

Epidemiology is study to clarify the outcome of a dose whose effect is not known well. Dose-Response test aims to clarify the positive (negative) correlation between an amount of dose and the outcome in a clinical laboratory test. Divided into several groups with same condition, the set of subjects are given a specified amount of doses for
Fig. 6 Processing time for dataset size in Bob

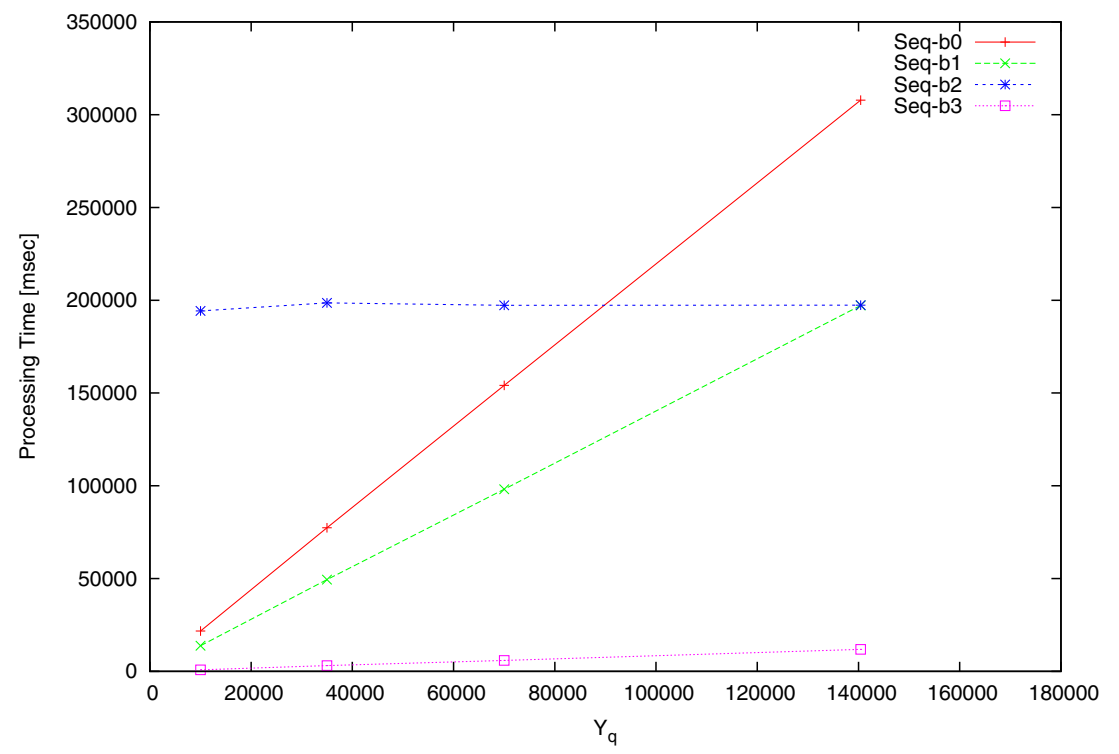


Table 7 Comparison of building blocks

\begin{tabular}{lllll}
\hline & \multicolumn{2}{l}{ AES03 [13] SSR [8] } & \multicolumn{2}{l}{ FNP04 [17] Proposed } \\
\hline Intersection & Available & \multicolumn{2}{l}{ No (size only) Available } & Available \\
Input form & Set & Vector & Set & Set \\
Complexity & $O(n)$ & $O(N)$ & $O\left(n^{2}\right)$ & $O(n)$ \\
Performance & - & $10 \mathrm{dim} / \mathrm{s}$ & - & 360 elements/s \\
Relative risk & N/A & N/A & N/A & Yes \\
Hypothesis test N/A & N/A & N/A & Yes \\
\hline
\end{tabular}

each group and observed the responses for the dose. The proposed protocol can be applied to privacy-preserving dose-response test in two parties.

\section{Big data study}

Link the database of individual tax payments from Tax and Customs office and educational records to reveal the correlations between working in university and their yearly income.

\section{Conclusions}

We have proposed a privacy-preserving hypothesis testing for epidemic studies for calculating relative risk of cancer from distributed providers. The proposed schemes allow independent provides to have confidential datasets to perform computing correlation between any interested attributes. Our experiment shows a close relative risk to conventional work with raw data, which indicating that the daily physical activities reduce a risk of cancer for some experiments in a significant level of confidence.

Acknowledgment We appreciate the support from Dr. Kawamura, Mr. Uozumi, Mr. Higashi, Mr. Koyanagi, Mr. Taguchi, Mr. Kato, and Mr. Ohkubo for giving insightful suggestions and cooperation for the experiments.

\section{Compliance with Ethical Standards}

Conflict of interests Hiroaki Kikuchi has received research grants from Cyber Communications Inc.

Ethical approval All procedures performed in studies involving human participants were in accordance with the ethical standards of the National Cancer Center.

Informed consent Informed consent was obtained from all individual participants included in the study.
Open Access This article is distributed under the terms of the Creative Commons Attribution 4.0 International License (http:// creativecommons.org/licenses/by/4.0/), which permits unrestricted use, distribution, and reproduction in any medium, provided you give appropriate credit to the original author(s) and the source, provide a link to the Creative Commons license, and indicate if changes were made.

\section{References}

1. Cardis, E., Vrijheid, M., Blettner, M., and Gilbert, E., Risk of cancer after low doses of ionizing radiation: retrospective cohort study in 15 countries, BMJ Online First, pp. 1-6, 2005.

2. Homer, N., Szelinger, S., and Redman, M., Resolving individuals contributing trace amounts of DNA to highly complex mixtures using high-density snp genotyping microarrays, Plos Genetics, 4(8), 2008

3. Smith, A., Privacy-preserving statistical estimation with optimal convergence rates, in proc. of the forty-third annual ACM symposium on Theory of computing pp. 813-822, 2011.

4. Agrawal, R., Evfimievski, A., and Srikant, R., Information sharing across private databases, in proc. of ACM SIGMOD International Conference on Management of Data, 2003.

5. Fienberg, S. E., Rinaldo, A., and Yang, X., Differential privacy and the risk-utility tradeoff for multi-dimensional contingency tables. In: Domingo-Ferrer, J., and Magkos, E. (Eds.) PSD 2010, LNCS 6344, pp. 187-199, 2010.

6. Liao, J. C., and Sankear, L., Hypothesis Testing in the High Privacy Limit, in proc. of 54th Annual Allerton Conference on Communication, Control, and Computing, pp. 649-656, 2016.

7. Kikuchi, H., Sato, T., and Sakuma, J., Privacy-Preserving Protocol for Epidemiology in Effect of Radiation, Proceedings of the 2013 Seventh International Conference on Innovative Mobile and Internet Services in Ubiquitous Computing (IMIS 13), pp. 831-836, IEEE, 2013.

8. Vaidya, J., and Clifton, C., Privacy preserving association rule mining in vertically partitioned data, in The Eighth ACM SIGKDD International Conference on Knowledge Discovery and Data Mining, SIGKDD, ACM Press, Edmonton, Canada, pp. 639-644, 2002.

9. Radiation Effects Association, Annual Report on radiation epidemiological study for workers in nuclear-power station, 2010. (written in Japanese).

10. Ministry of Health, Labor and Welfare, the Vital Statistics of Japan. (available from http://www.mhlw.go.jp/english/database/ index.html).

11. Yasui, R., Sato, K., Harigaya, T., Kanai, A., Hirota, K., and Tanimoto, S., A proposal of privacy search oracle model for estimating personal information disclosure level of blog articles. IPSJ SIG Technical Report 2009-EIP-43:9-16, 2009. (in Japanese).

12. Pagano, M., and Gauvreau, K., Principles of biostatistics 2nd ed., Brooks/Cole, 2000.

13. Agrawal, R., Evfimievski, A., and Srikant, R., Information sharing across private databases, in proc. of ACM SIGMOD International Conference on Management of Data, 2003.

14. Paillier, P., Public-key cryptosystems based on composite degree residuosity classes, In Advances In Cryptology - Eurocrypt 1999, pp. 223-238, Springer, 1999.

15. Pohlig, S., and Hellman, M., An Improved Algorithm for Computing Logarithms over GF(p) and its Cryptographic Significance, IEEE Transactions on Information Theory, (24), pp. 106110, 1978.

16. Goethals, B., Laur, S., Lipmaa, H., and Mielikainen, T., On Secure Scalar Product Computation for Privacy-Preserving Data Mining, 
In Choonsik Park and Seongtaek Chee, editors, The 7th Annual International Conference in Information Security and Cryptology (ICISC 2004), volume 3506, pp. 104-120, December 2.3, 2004.

17. Freedman, M. J., Nissim, K., and Pinkas, B., Efficient private matching and set intersection, EUROCRYPT 2004, LNCS 3027 , pp. 1-19, Springer, 2004.

18. Abadi, A., Terzis, S., Metere, R., and Dong, C., Efficient Delegated Private Set Intersection on Outsourced Private Datasets,
Proceedings of the 30th International Conference on ICT Systems Security and Privacy Protections (SEC 2015), pp. 3-17, 2015.

19. Inoue, Daily total physical activity level and total cancer risk in men and women: results from a large-scale population-based cohort study in Japan. Am. J. Epiderniol. 168:391-403, 2008.

20. Tanaka, Y., Frequency of people with same first and last names. IPSJ SIG Technical Report on Natural Language (NL) 1977-NL010:1-7, 1977. 